\title{
Highly Efficient Visible Blue-Emitting Black Phosphorus Quantum Dot: Mussel-Inspired Surface Functionalization for Bioapplications
}

Miyeon Lee, ${ }^{\dagger} \bigcirc$ Young Ho Park, ${ }^{\ddagger}, \bigcirc$ Eun Bi Kang, ${ }^{\S}$ Ari Chae, " Yujin Choi," Seongho Jo," Yu Jin Kim," Soo-Jin Park, ${ }^{\nabla} \odot$ Byunggak Min,,$"$ Tae Kyu An, Jihoon Lee, ${ }^{\| \odot ~ S u-I l ~ I n, ~ S a n g ~ Y o u l ~ K i m, ~}{ }^{\ddagger}, \oplus$ Sung Young Park, $*, \S, \perp_{\mathbb{C}}$ and Insik In*, $\|_{1}, \perp_{\mathbb{C}}$

${ }^{\dagger}$ Department of Chemistry, KAIST, Yuseong-gu, Daejeon 34141, South Korea

${ }^{\ddagger}$ Department of Energy System Engineering, DGIST, Daegu 42988, South Korea

${ }^{\S}$ Department of Chemical \& Biological Engineering, ${ }^{\|}$Department of Polymer Science and Engineering, and ${ }^{\perp}$ Department of IT Convergence (Brain Korea PLUS 21), Korea National University of Transportation, 50 Daehak-ro, Chungju-si, Chungbuk 27909, South Korea

${ }^{\#}$ Center for Nanoscale Materials, Argonne National Laboratory, Lemont, Illinois 60439, United States

${ }^{\nabla}$ Department of Chemistry, Inha University, Incheon 402-751, South Korea

\section{Supporting Information}

ABSTRACT: The preparation of blue-emitting black phosphorus quantum dots (BPQDs) is based on the liquid-phase exfoliation of bulk BP. We report the synthesis of soluble $\mathrm{BPQDs}$ showing a strong visible blue-light emission. Highly fluorescent (photoluminescence quantum yield of $\approx 5 \%$ with the maximum emission $\left(\lambda_{\max }\right)$ at $\left.\approx 437 \mathrm{~nm}\right)$ and dispersible BPQDs in various organic solvents are first prepared by simple ultrasonication of BP crystals in chloroform in the ambient atmosphere. Furthermore, simple mussel-inspired surface functionalization of BPQDs with catechol-grafted poly(ethylene glycol) in basic buffer afforded water-soluble blue-emitting $\mathrm{BPQDs}$ showing long-term fluorescence stability, very low cytotoxicity, and excellent fluorescence live cell imaging capability.

\section{INTRODUCTION}

Elemental nanomaterials based on group IV elements such as carbon, $^{1-3}$ silicon, ${ }^{4-6}$ and germanium ${ }^{7,8}$ have received significant research interests owing to their unique properties applicable to various promising applications. Graphene, a twodimensional (2D) crystal made of $\mathrm{sp}^{2}$-hybridized carbon atoms, has been widely investigated owing to its fascinating optoelectrical and mechanochemical properties. ${ }^{9,10}$ Recently, another elemental nanomaterial based on phosphorus, a group $\mathrm{V}$ element, has emerged as the new 2D layered material.

Similar to graphene, phosphorene, a single layer or few layers of black phosphorus (BP), has been isolated through the mechanical exfoliation from bulk BP and has immediately received tremendous attention owing to its remarkable optoelectronic performance. ${ }^{18,19}$ For example, phosphorenebased nanodevices have demonstrated the drain current modulation and carrier mobility up to $10^{5}$ and $1000 \mathrm{~cm}^{2} /(\mathrm{V}$. $\mathrm{s})$, respectively, showing the potential of phosphorene as a new 2D material applicable in nanoelectronic devices. ${ }^{20,21}$ In addition, a few-layer phosphorene demonstrates direct and highly layer-dependent photoluminescence (PL) through tuning band gaps from mid-infrared to near-infrared wavelengths, showing promise for the application in high-performance optoelectronic devices. ${ }^{22,23}$

Very recently, few studies have shown the facile preparation of soluble $\mathrm{BP}$ or phosphorene quantum dot (QD) by the liquid-phase exfoliation of BP crystal, which is beneficial for the wider utilization of the intriguing properties of phosphorene through various wet processes. ${ }^{24-26}$ Despite the recent rapid progress in the solution-based methods of BP quantum dots (BPQDs), it is still a great challenge to prepare soluble BPQDs with unexplored optical properties such as strong fluorescence in the visible wavelength ranges and to explore the florescent BPQDs in bioapplications such as physiologically nontoxic fluorescence bioimaging agents.

In the present study, highly blue-emitting BPQDs with photoluminescence quantum yield (PLQY) of $\approx 5 \%$, fluorescence stability, and long-term dispersion in various organic solvents are first prepared by simple ultrasonication of BP in chloroform in the ambient atmosphere. Furthermore, the mussel-inspired surface functionalization and the subsequent solubility switching of BPQDs were successfully performed with the utilization of catechol-grafted poly(ethylene glycol) (CA-PEG) in basic buffer, resulting in water-soluble and blueemitting PEG-modified BPQD (PEG-BPQD) showing both very low cytotoxicity and fluorescence cell imaging ability.

Received: July 25, 2017

Accepted: October 11, 2017

Published: October 23, 2017 
(a)

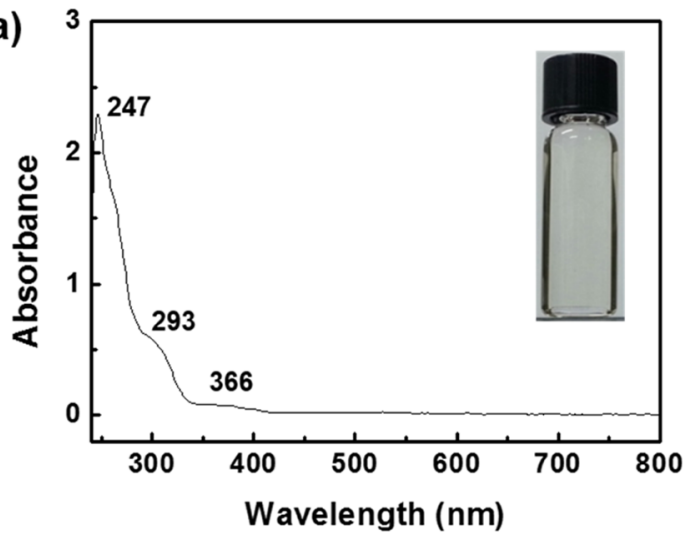

(c)

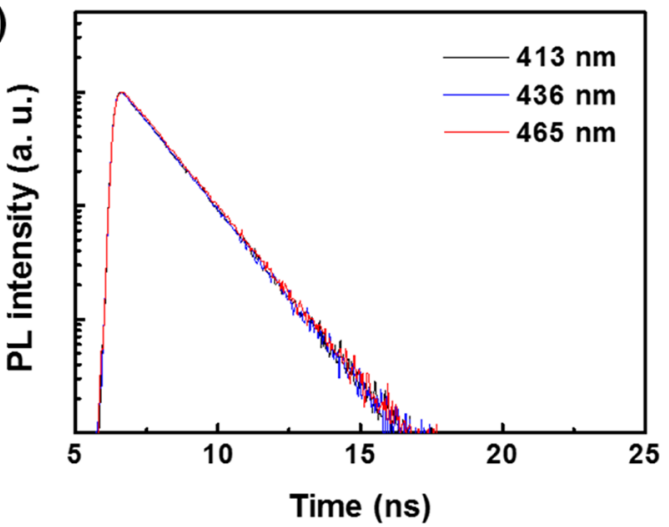

(b)

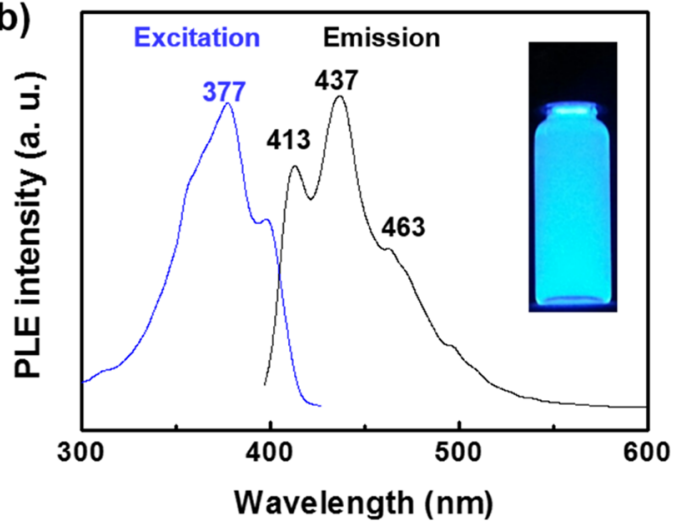

(d)

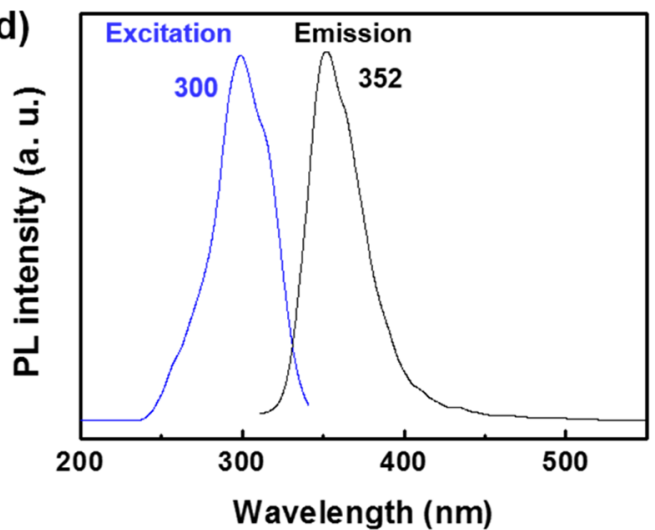

(e)

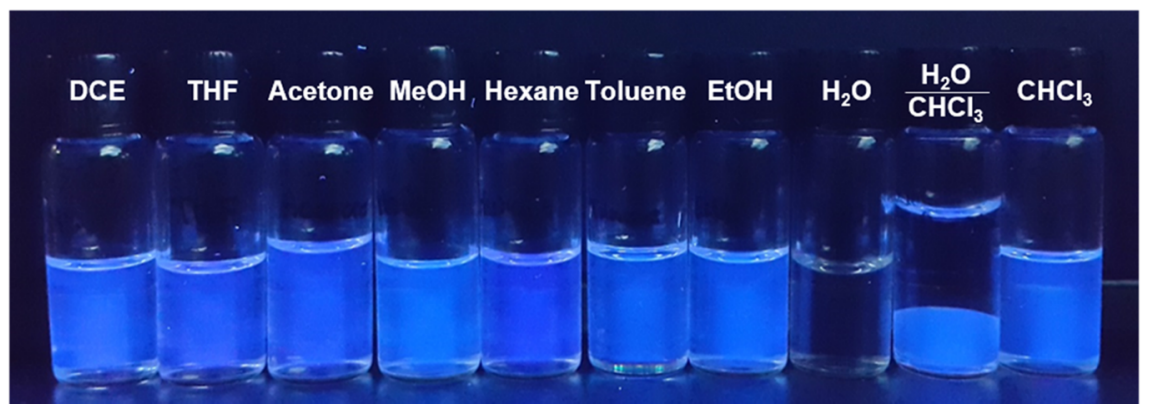

Figure 1. (a) UV-vis spectrum of the chloroform dispersion of the BPQDs. (b) PL and PLE spectra of the blue-emitting BPQDs. (c) The luminescence decay profiles of the blue-emitting BPQDs dispersion prepared in chloroform. (d) The PLE and PL spectra showing that the UV emission at $352 \mathrm{~nm}$ is maximized at the excitation wavelength of $300 \mathrm{~nm}$. (e) Photo images of the dispersions of blue-emitting BPQDs in various solvents.

\section{RESULTS AND DISCUSSION}

Twenty-four organic solvents in various solvent categories with different surface tensions and molecular weights were tested toward the liquid-phase exfoliation of BP flake (the Supporting Information (SI), Table S1). Ultrasonication was attempted to break down the van der Waals interactions among the strong BP interlayers, similarly to the liquid-phase exfoliation of graphite and boron nitride to prepare single- or few-layered graphene and boron nitride nanosheets. ${ }^{27-30}$ To minimize the effects of other experimental parameters such as temperature and humidity, all of the liquid-phase exfoliation setups were attempted simultaneously using a tip-sonicator attached with a 24 multitip accessory. After ultrasonication for $1 \mathrm{~h}$, nonexfoliated bigger BP particles were removed from the dispersion of the BPQDs by centrifugation at either 2500 or $7500 \mathrm{rpm}$. Then, the PL emission of each dispersion was examined by irradiation using a hand-held ultraviolet lamp with
$365 \mathrm{~nm}$ wavelength. Surprisingly, among the 24 organic solvents, medium to strong blue emissions were observed in $\mathrm{N}, \mathrm{N}$-dimethylacetamide (DMAc), pyridine, and chloroform. The degree of exfoliation, identified by monitoring the ultraviolet visible (UV-vis) spectrum of each dispersion, did not sturdily correlate to the observation of the blue emission. For example, alcoholic solvents such as methanol, ethanol, isopropanol, and ethylene glycol did not show reasonable effectiveness both for exfoliation and PL emission, whereas chloroform and pyridine showed weak exfoliation performance but strong blue and green PL emission, respectively. In the case of aprotic polar solvents, reported to be a choice of organic solvents for the liquid-phase exfoliation of graphite and even $\mathrm{BP},{ }^{31,32,24}$ blue emission was observed in DMAc, whereas $\mathrm{N}$ methylpyrrolidone (NMP) and $\mathrm{N}, \mathrm{N}$-dimethylformamide (DMF) were only effective for the exfoliation itself. 
(a)
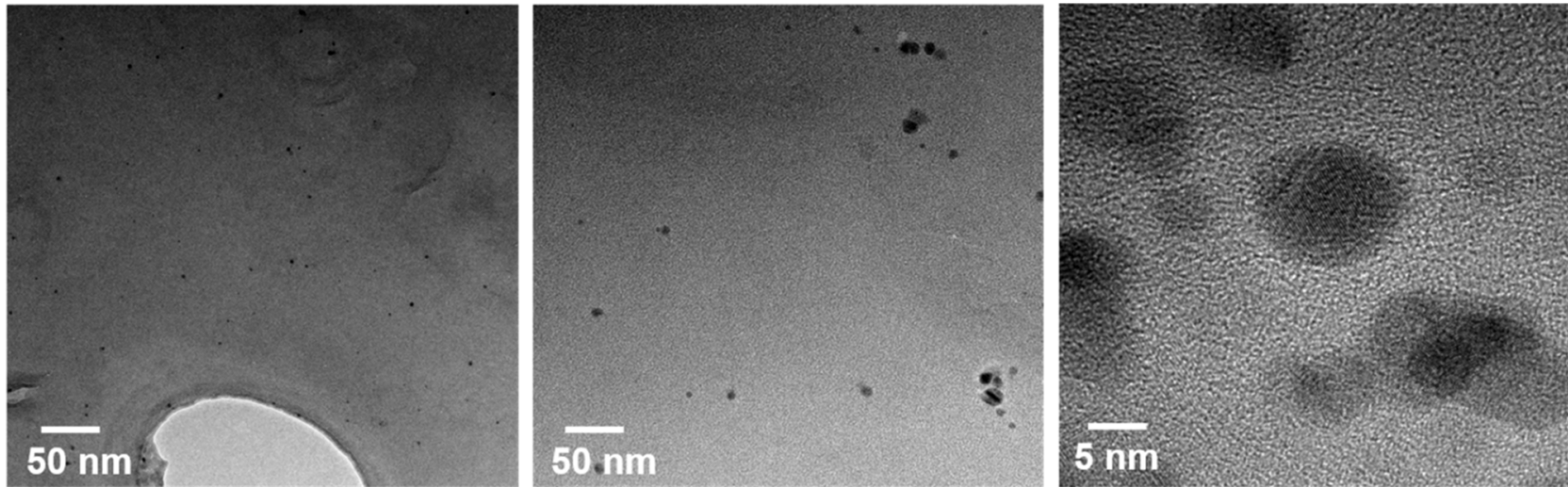

(b)
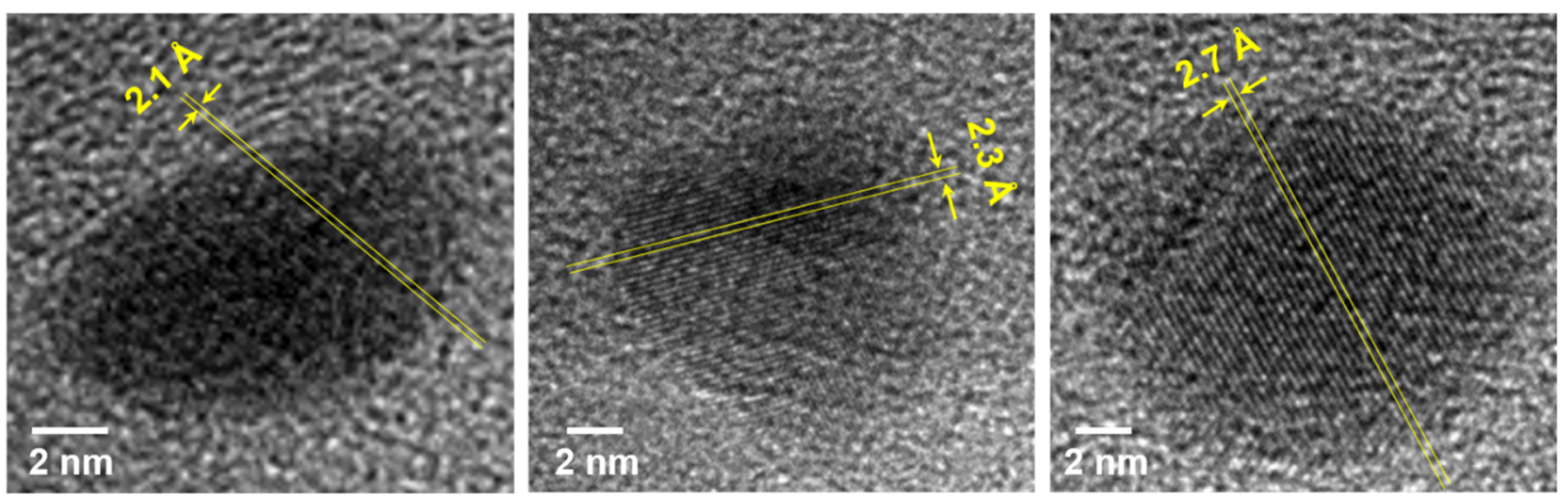

(c)

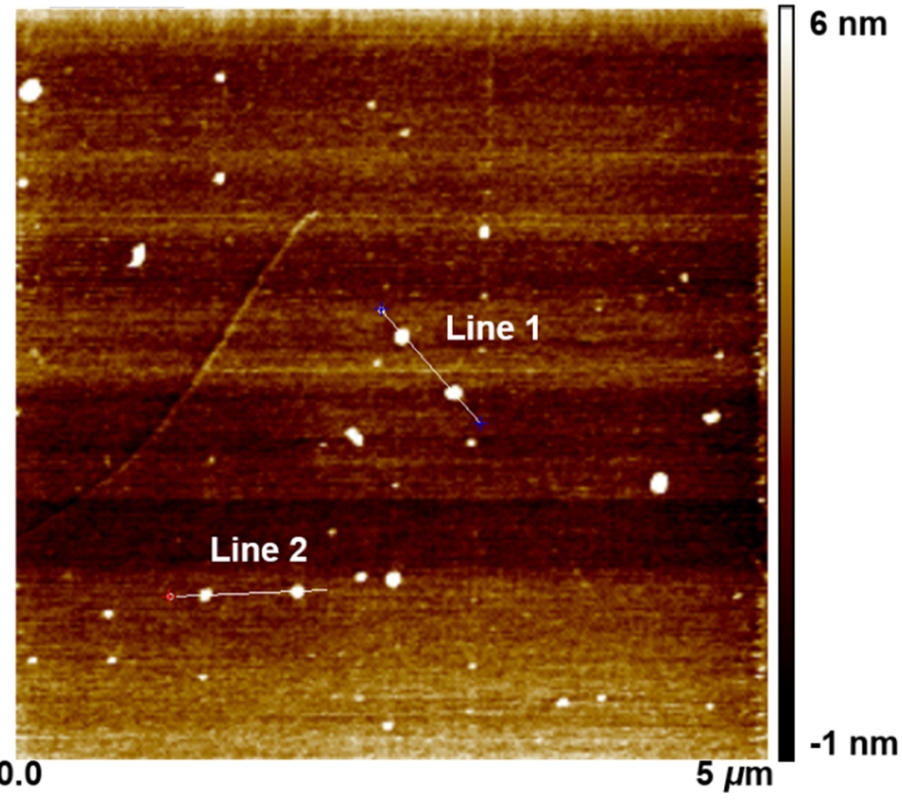

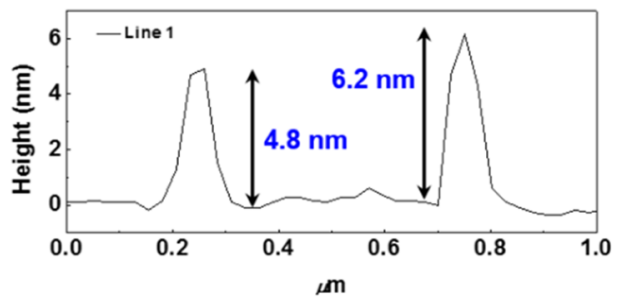

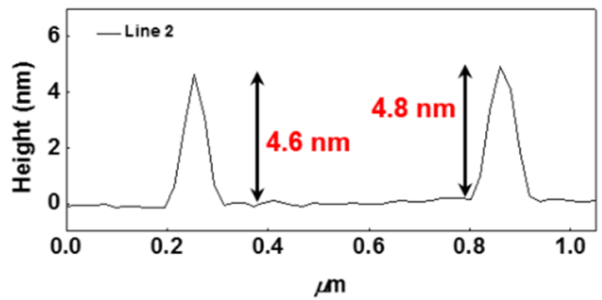

$\mathrm{nm}$

Figure 2. (a) TEM images of BPQDs. (b) The HRTEM images of BPQDs. (c) The AFM topographic images and sectional view of BPQDs.

In the case of chloroform, the UV-vis spectrum of the chloroform dispersion of the BPQDs showed three overlapped optical absorption peaks at 247, 293, and $366 \mathrm{~nm}$ (5.03, 4.23, and $3.39 \mathrm{eV}$ ), respectively (Figure 1a). The highest PL emission intensity was observed for the BPQD dispersion in chloroform at $437 \mathrm{~nm}$ with an excitation wavelength of $377 \mathrm{~nm}$, and the position of the maximum emission peak did not show any noticeable shift regardless of the excitation wavelength (Figure S1). The strong PL emission peak between 400 and $550 \mathrm{~nm}$ is regarded to be composed of three major peaks overlapping each other at 413,437, and $463 \mathrm{~nm}$ (3.00, 2.84, and $2.68 \mathrm{eV}$ ), respectively. However, the full width at halfmaximum (FWHM) height of this emission peak is as small as $60 \mathrm{~nm}$, with an excitation wavelength of $377 \mathrm{~nm}$. Even though the FWHM of blue-emitting BPQDs is not close to the smallest FWHM $(\approx 20 \mathrm{~nm})$ of Cd- or Pb-based inorganic QDs, it is much smaller than the FWHM of carbon quantum dots (CQDs) or carbon dots (CDs) (typically $>100 \mathrm{~nm}$ ) and silicon quantum dot (typically $>70 \mathrm{~nm}) .^{33-36}$ The photoluminescence excitation (PLE) spectra clearly demonstrate that the observed overlapping luminescence peak centered at $437 \mathrm{~nm}$ of the blueemitting BPQDs could be correlated to the three new 
(a)

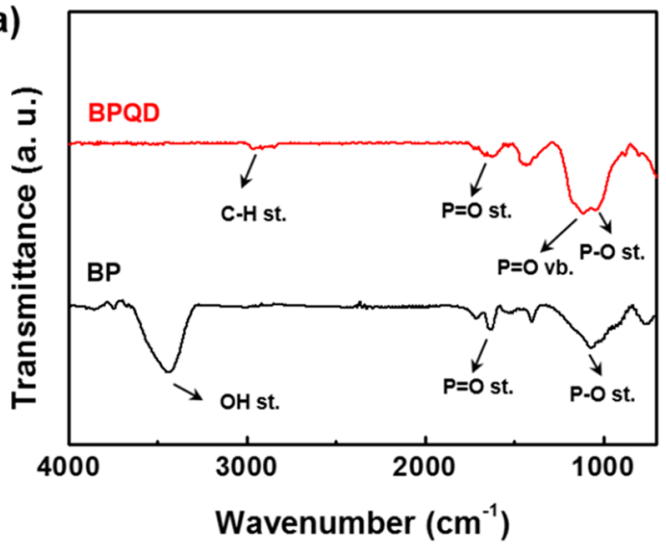

(c)

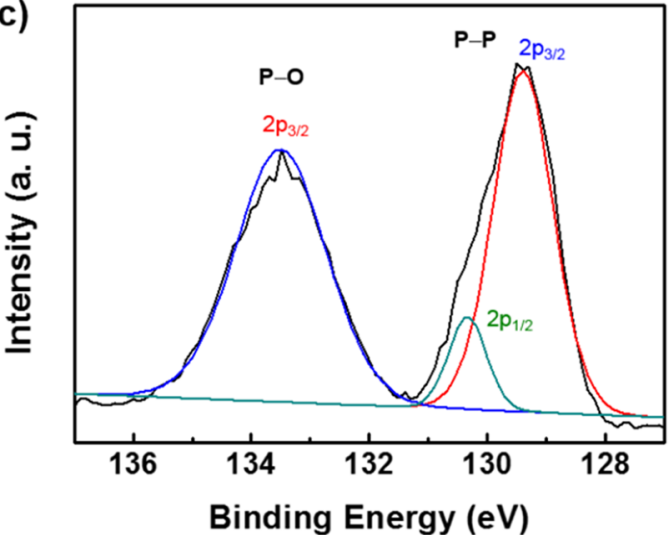

(e) (b)

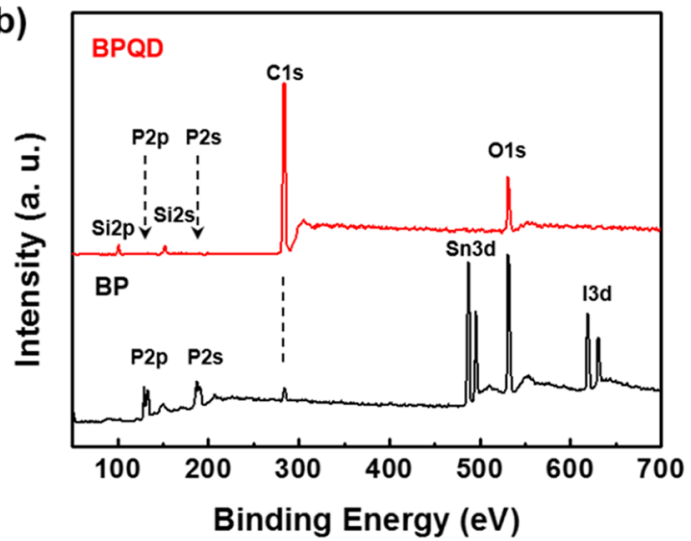

(d)

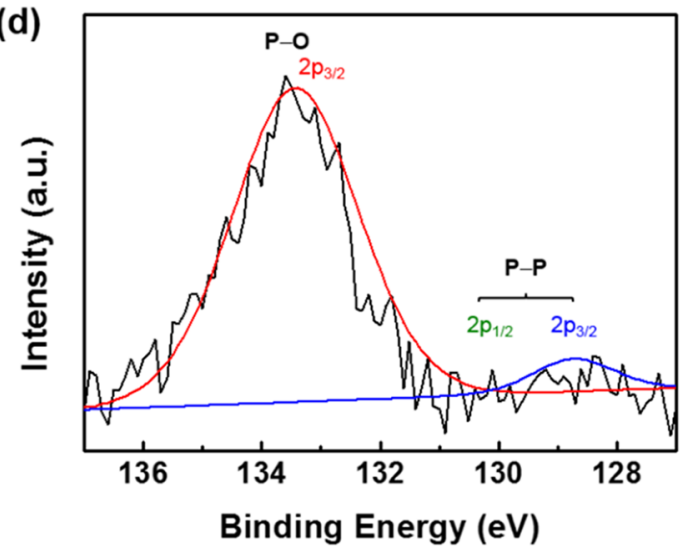

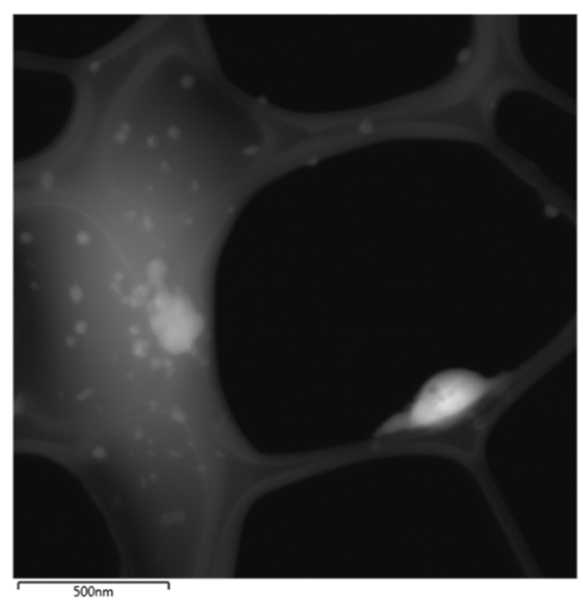
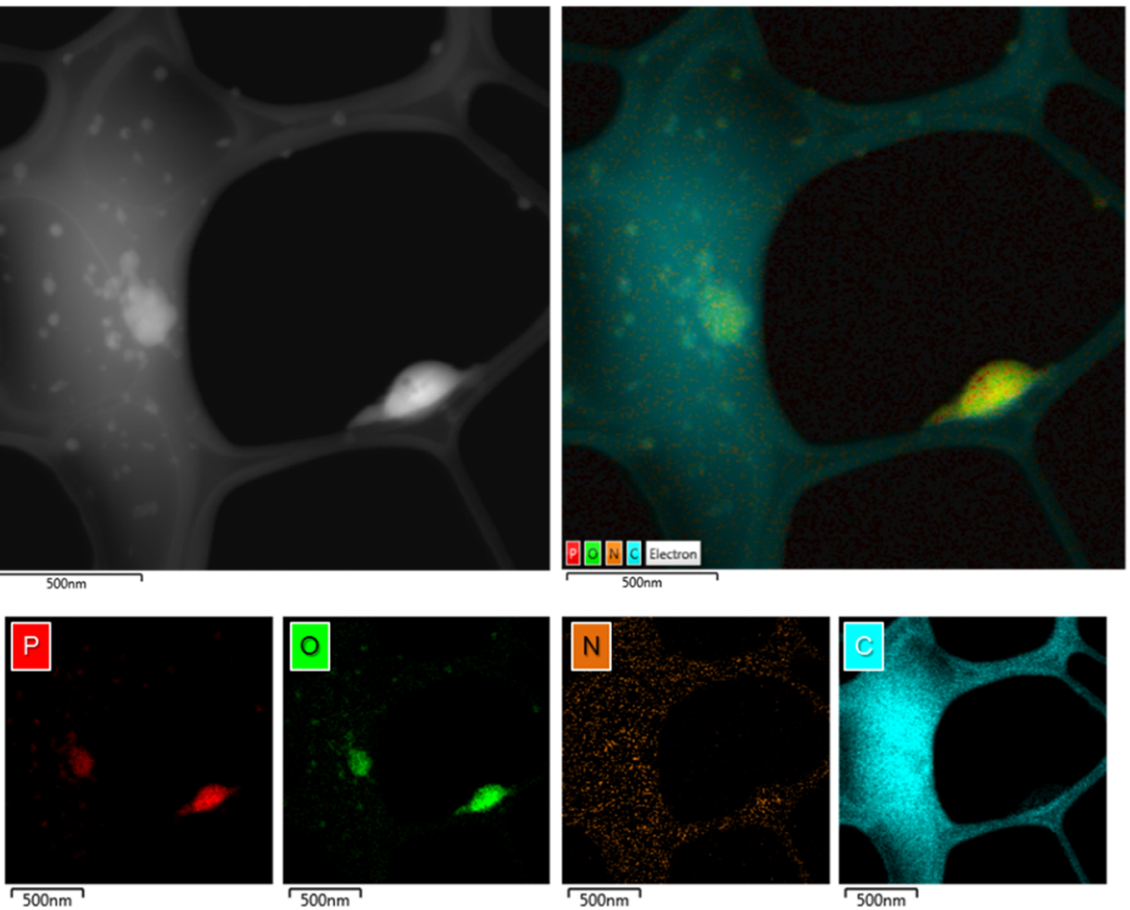

Figure 3. (a) FTIR spectra of BPs and BPQDs. (b) XPS survey scans of BPs and (c) high-resolution P 2p spectrum of the pristine BPs. (d) Highresolution P $2 p$ spectrum of BPQDs. (e) EDX mapping of the TEM image of the BPQDs.

electronic transitions at 364, 377, and $398 \mathrm{~nm}(3.41,3.28$, and $3.12 \mathrm{eV}$ ), respectively (Figure $1 \mathrm{~b})$. The luminescence decay profiles of the blue-emitting BPQDs dispersion prepared in chloroform were recorded for the transitions at 413,437 , and $463 \mathrm{~nm}$ at the excitation wavelength of $375 \mathrm{~nm}$ at room temperature by time-correlated single-photon counting techni- que (Figure 1c). Regardless of the wavelength of the transition, the decay profiles of the blue-emitting BPQDs very well fitted to the single-exponential decays with the same lifetime $\left(\tau_{1}\right)$ of $1.75 \mathrm{~ns}$, indicating that the blue emission from the BPQDs prepared in chloroform might be originating from the bandedge states rather than the trap states, which is also supported 
by the excitation-independent emission property of the BPQDs. ${ }^{11}$

In addition to the strong blue emission at $437 \mathrm{~nm}$, the BPQDs prepared in chloroform showed an additional feature in their PL emission spectra. First, the as-prepared BPQDs showed weak UV emission at $352 \mathrm{~nm}(3.52 \mathrm{eV})$ with a FWHM of $39 \mathrm{~nm}$ upon irradiation of UV ranging from 250 to $325 \mathrm{~nm}$. The PLE spectrum shows that the UV emission at $352 \mathrm{~nm}$ is maximized at the excitation wavelength of $300 \mathrm{~nm}(4.13 \mathrm{eV})$ (Figure 1d). Typically, the pristine BP is known to be easily oxidized by the exposure to ambient atmosphere within few hours. ${ }^{37-40}$ Therefore, significant oxidation of the prepared BPQDs is expected, but ${ }^{24}$ the blue or UV emission of the BPQDs prepared in chloroform did not show any noticeable decrease in the emission intensity even after 1 month. The pristine BP layers are reported to show a layer-dependent direct band gap ranging from $\approx 0.3 \mathrm{eV}$ in bulk to $>1 \mathrm{eV}$ in monolayer. ${ }^{41-43}$ However, the absorption and emission behaviors of the BPQDs in this study show that the BPQDs prepared in chloroform has completely different electronic structures compared to the previous reports, indicating that the BPQDs might have different morphological or compositional features. The blue-emitting BPQDs prepared in chloroform were highly dispersible in organic solvents, including toluene and methanol, regardless of the polarity, but completely insoluble in water (Figure 1e). No significant change in both the emission intensity and maximum emission wavelength was observed. Mixing of the chloroform dispersion of BPQD with water instantly induced the phase separation; however, the bottom chloroform BPQD dispersion did not lose its PL intensity even after several weeks, indicating the resistance of the blue-emitting BPQDs for the hydrolysis or oxidation by excess water and ambient oxygen.

Similarly, irradiation-independent PL emission centered at $488 \mathrm{~nm}(2.54 \mathrm{eV})$ was observed when DMAc was used as the organic solvent during the liquid-phase exfoliation of BP; however, the PL intensity was much weaker than in the case of chloroform (the SI, Figure S2). Interestingly, in pyridine, irradiation-dependent PL emission with maximum emission wavelength ranging from $395 \mathrm{~nm}$ (325 nm excitation) to 442 $\mathrm{nm}$ (375 nm excitation) and further to $511 \mathrm{~nm}(450 \mathrm{~nm}$ excitation) was observed similarly to the PL emission behavior of certain CQDs or CDs showing tunable fluorescence emissions through the surface defect-derived origins (the SI, Figure S3). ${ }^{44,45}$ Therefore, fluorescence emission mechanism of the BPQDs prepared by the liquid-phase exfoliation of BP is roughly estimated to originate from the band gap transitions in the cases of chloroform and DMAc or the radiative recombination of the surface-confined electrons and holes in the case of pyridine.

BPQDs prepared in chloroform were investigated by both the transmission electron microscopy (TEM) and atomic force microscopy (AFM). The TEM images show the BPQDs with an average size of $5.9 \pm 4.4 \mathrm{~nm}$ after checking $20 \mathrm{BPQD}$ particles (Figure 2a). The high-resolution TEM (HRTEM) images of the BPQDs indicate $d$-spacings of $0.21,0.23$, and 0.27 $\AA$, which were assigned to the (131), (141), and (040) planes, respectively, consistent with the expected crystal structure of BP crystal (Figure 2b). ${ }^{46}$ The AFM topographic images of the exfoliated blue-emitting BPQDs showed the presence of a range of shapes and sizes of the BPQDs. The measured heights ranged from 4.6 to $6.2 \mathrm{~nm}$, corresponding to the BPQDs with approximately $8-12$ layers (Figure 2c). ${ }^{24}$ The lateral dimensions of these thin BPQD particles are relatively wider than those observed by the TEM analysis, probably owing to the presence of lateral aggregation of several BPQDs on a $\mathrm{Si} /$ $\mathrm{SiO}_{2}$ substrate during the sample preparation. Raman spectroscopy was used to analyze the few-layered BPQDs prepared by the liquid-phase exfoliation of BP crystal in chloroform. The blue-emitting BPQDs showed characteristic Raman peaks at 362.6, 440.0, and $467.0 \mathrm{~cm}^{-1}$, attributed to one out-of-plane phonon mode $\left(A_{1 g}\right)$ and two in-plane modes $\left(B_{2 g}\right.$ and $\left.A_{2 g}\right)$, whereas the pristine $\mathrm{BP}$ flake showed the corresponding Raman peaks at $363.8,439.2$, and $466.7 \mathrm{~cm}^{-1}$ (the SI, Figure S4). ${ }^{47}$ The preservation of the Raman peak positions of BP in BPQD indicates that the BPQDs retain the crystalline lattice of $\mathrm{BP}$ even after the liquid-phase exfoliation in chloroform. Although there might be further oxidation of BP layer(s) by atmospheric oxygen, the BPQD dispersion definitely displayed unaffected blue emission regardless of the prolonged storage in ambient atmosphere without special protection such as nitrogen purging.

As BP flakes often present environmental instability toward atmospheric oxygen and water because of the easy oxidation or hydrolysis of the pristine $\mathrm{BP}$, the precise compositional features of the prepared BPQDs should be investigated carefully to fully explore the fluorescent BPQD dispersion as new nanomaterials for optoelectrical or biological applications. Fourier transform infrared (FTIR) spectroscopy, X-ray photoelectron spectroscopy (XPS), and energy-dispersive X-ray spectroscopy (EDX) analysis were performed for both the pristine BP and BPQD. The FTIR spectrum of BPQD showed strong peaks at 1661, $1429,1110,1045$, and $878 \mathrm{~cm}^{-1}$ (Figure 3a). The peaks at 1661 and $1110 \mathrm{~cm}^{-1}$ are assigned to the $\mathrm{P}=\mathrm{O}$ stretching and vibrational modes, respectively, and the peaks at 1045 and 878 $\mathrm{cm}^{-1}$ are assigned to the $\mathrm{P}-\mathrm{O}$ stretching modes. The pristine BP also showed a very characteristic set of peaks at 3439,1642 , 1401,1065 , and $760 \mathrm{~cm}^{-1}$ in its FTIR spectrum. The peak at $3439 \mathrm{~cm}^{-1}$ is attributed to the $\mathrm{O}-\mathrm{H}$ stretching mode, and the peak at $1642 \mathrm{~cm}^{-1}$ is assigned to the $\mathrm{P}=\mathrm{O}$ stretching mode. ${ }^{48}$ The broad peak centered at $\sim 1065 \mathrm{~cm}^{-1}$ was ascribed to the $\mathrm{P}-\mathrm{O}$ stretching mode. The presence of those peaks in BP supports the fact that the pristine $\mathrm{BP}$ is partially oxidized even before solvent exfoliation by ambient moisture. However, the $\mathrm{O}-\mathrm{H}$ stretching peak of $\mathrm{BP}$ was not observed in BPQD, even though all of the other peaks were observed, describing that certain chemical reaction occurred during the liquid-phase exfoliation of BP in chloroform. The most plausible explanation is that certain alkylation reaction occurred, resulting in the formation of self-passivation layers on the surface of BPQDs and the subsequent retardation of further oxidation of BPQDs. As previously mentioned, the prepared BPQDs were easily dispersible even in highly nonpolar organic solvents such as toluene and hexane, but insoluble in water. Moreover, small peak(s) was observed at $\sim 2916 \mathrm{~cm}^{-1}$ in the FTIR spectrum of $\mathrm{BPQD}$, which corresponds to the $\mathrm{C}-\mathrm{H}$ stretching mode of the alkyl groups. The hydrophobic nature of BPQDs facilitates the preparation of hydrophobic polymer/BPQD composite films by simple mixing in common organic solvents. Fluorescent polystyrene (PS)/BPQD composite film was simply prepared by this approach (the SI, Figure S5). 2 wt \% BPQD-included PS/BPQD composite film demonstrated a strong blue fluorescence under UV irradiation. Fluorescence spectra of the composite film retained the unique fluorescence profile of pristine BPQDs (the SI, Figure S6). Excitation with 300 or 377 $\mathrm{nm}$ light resulted in the maximum fluorescence intensities at 

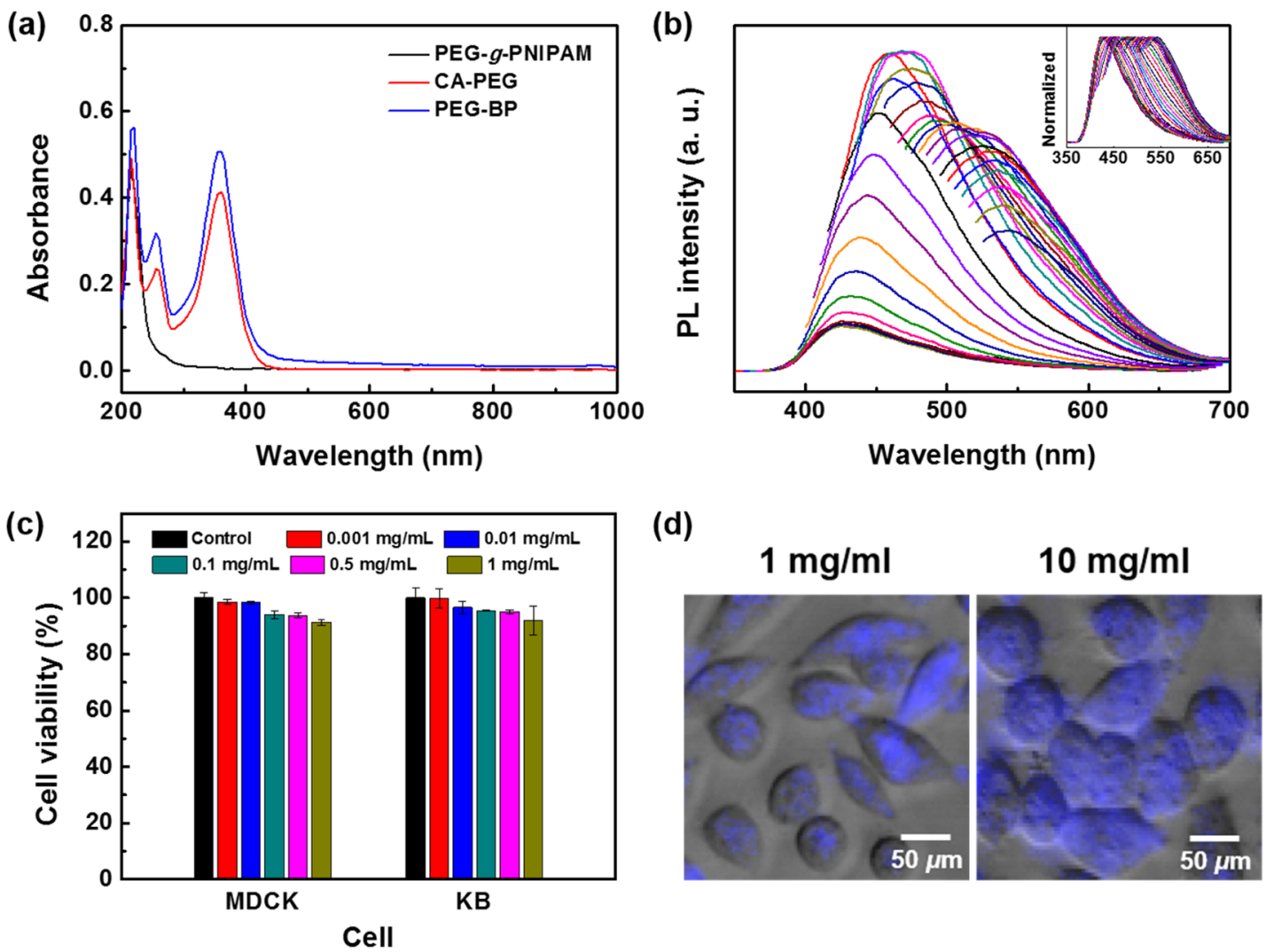

(d)
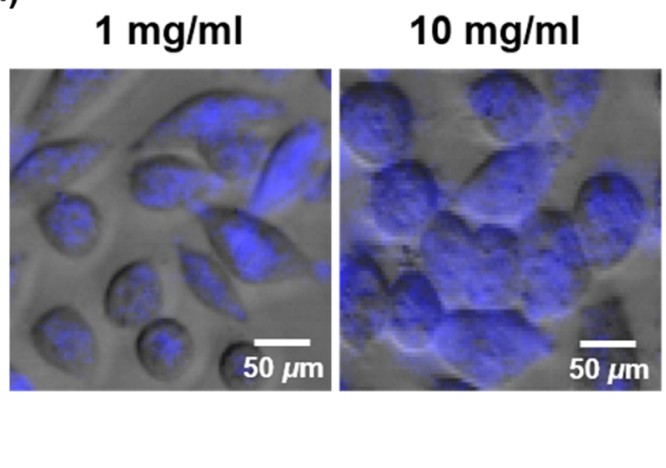

Figure 4. (a) UV-vis absorption spectra of PEG-g-PNIPAM (black line), CA-PEG (red line), and PEG-BPQD (blue line). (b) PL spectra and normalized PL spectra (top-right inset) of the PEG-BPQDs. (c) In vitro cell viability of PEG-BPQD investigated toward either MDCK normal cells or KB tumor cells depending on the concentration of BPQDs. (d) Confocal fluorescence image of KB tumor cells treated with PEG-BPQDs (1 or 10 $\mathrm{mg} / \mathrm{mL}$, respectively).

345 (UV emission) and 409/434.5 nm (blue emission), respectively, which closely match the fluorescence behavior of BPQDs.

All of the above results show that BPQDs have significantly oxidized phosphorous atoms in the forms of $\mathrm{P}-\mathrm{O}-\mathrm{C}$ probably near the surface of the BPQD particles. The XPS survey of the BPQDs shows binding peaks for P $2 \mathrm{p}$ and $\mathrm{P} 2 \mathrm{~s}$ at 131.6 and $189.2 \mathrm{eV}$, respectively, together with $\mathrm{O} 1 \mathrm{~s}$ peak at $530.1 \mathrm{eV}$ and $\mathrm{C} 1 \mathrm{~s}$ peak at $283.1 \mathrm{eV}$ (Figure $3 \mathrm{~b}$ ). The high-resolution P 2p spectrum shows a peak in the high-energy region at $133.5 \mathrm{eV}$, which again shows the high oxidation state $(\mathrm{P}-\mathrm{O})$ of the $\mathrm{BPQDs}$, and a small peak at $128.7 \mathrm{eV}$ ( $\mathrm{P}-\mathrm{P}$ binding peak) (Figure $3 \mathrm{~d}$ ), whereas the pristine BP shows a higher intensity for the $\mathrm{P}-\mathrm{P}$ binding peak than that of the $\mathrm{P}-\mathrm{O}$ binding peak (Figure 3c). The EDX mapping of the TEM image of the BPQDs definitely revealed significant $\mathrm{P}$ and $\mathrm{O}$ atoms in both the isolated or aggregated BPQDs (Figure 3e). In the TEM EDX mapping image, oxygen atoms were found to distribute over the entire BPQD particles, and therefore a clear buildup of $(\mathrm{R}-\mathrm{O})_{x}-\mathrm{P} @ \mathrm{P}$ core-shell structure of the BPQDs, where $\mathrm{R}$ is methyl, is not reasonable in this stage. ${ }^{29}$

All of the above experimental evidences clearly show that $\mathrm{BPQDs}$ have rich oxidized phosphorous atoms either on the surface or throughout the BPQDs. If either $\mathrm{P}-\mathrm{OH}$ or $\mathrm{P}=\mathrm{O}-$ $\mathrm{OH}$ groups are located at the edge of the $\mathrm{BP}$ layer of the BPQDs as dangling bonds, exfoliated BPQDs should provide negative $\zeta$ potential values. However, positive $\zeta$ potential values of more than $+70 \mathrm{mV}$ was repeatedly observed for the chloroform dispersion of the BPQDs (the SI, Figure S7). The insolubility of the BPQDs into aqueous media also correlates to this observation because the presence of $\mathrm{P}-\mathrm{OH}$ or $\mathrm{P}=\mathrm{O}-\mathrm{OH}$ group should have enhanced the dispersion of BPQDs in aqueous media. To obtain further detailed information on the surface functionalities of BPQDs, phosphorus nuclear magnetic resonance $\left({ }^{31} \mathrm{P}\right.$ NMR) spectroscopy was recorded for both the pristine $\mathrm{BP}$ flakes (solid NMR) and $\mathrm{BPQD}$ dispersion in deuterated chloroform $\left(\mathrm{CDCl}_{3}\right)$, with $85 \%$ phosphoric acid as the reference. The chemical shift of the reference was $0 \mathrm{ppm}$ (the SI, Figure S8). BPQD showed two distinct phosphorus peaks at -13.1 and $-19.8 \mathrm{ppm}$, whereas the pristine BP showed a single peak at 19.9 ppm. ${ }^{49}$ The distinct chemical shift of phosphorous atoms in BPQD is regarded to originate from two factors. One is nearly complete oxidation of $\mathrm{P}$ atoms in BPQD, and another is the chemical reaction between chloroform and the $\mathrm{P}-\mathrm{O}$ moieties of BPQDs. We assumed that the alkylation of the $\mathrm{P}-\mathrm{O}$ moieties of BPs or BPQDs by chloroform, the solvent itself, during the liquid-phase exfoliation step provides organo-soluble BPQDs. The ${ }^{13} \mathrm{C}$ NMR spectrum of BPQD also clearly shows the presence of carbon peaks at 29.9 and $27.1 \mathrm{ppm}$, supporting the presence of $(\mathrm{R}-\mathrm{O})_{x}-\mathrm{P}$ moieties in $\mathrm{BPQDs}$, where $\mathrm{R}$ is originating from the alkyl group of chlorinated solvent, chloroform, in this study (the SI, Figure S9). Further investigation is underway to fully understand the alkylation reaction of BPs in halogenated organic solvents during sonication-assisted exfoliation of BPs.

The blue emission of the BPQDs might be beneficial for designing BP-based nanomaterials as bioimaging agents. However, the as-formed BPQDs cannot be utilized for the fluorescence bioimaging because BPQD is completely insoluble in the aqueous media. Therefore, a facile surface modification 
method of BPQDs is urgently required. Therefore, musselinspired surface engineering of BPQDs was attempted by using catechol-grafted hydrophilic polymer because the surface functionalization proceeded by the catechol chemistry is widely acceptable on various nanomaterials with different compositions and morphologies. ${ }^{50-52}$ At first, aqueous soluble catecholgrafted PEG (CA-PEG) was prepared by the reaction between 2-chloro-3', $4^{\prime}$-dihydroxyacetophenone (CCDP) and poly $(\mathrm{N}$ isopropyl acrylamide)-grafted poly(ethylene glycol) (PEG-gPNIPAM) by the quaternization reaction of CCDP on the nitrogen moieties of PNIPAM. The number of catechol groups in single CA-PEG polymer was estimated as 15 from the ${ }^{1} \mathrm{H}$ NMR spectrum of the prepared CA-PEG (the SI, Figure S10). ${ }^{53}$ At second, the mussel-inspired surface functionalization of BPQDs with CA-PEG was attempted in a mixed solvent of EtOH and tris-buffered silane (TBS, $\mathrm{pH}$ 9.0). After purification by centrifugation and freeze drying, the PEG-modified BPQD (PEG-BPQD) was successfully obtained with $>90 \%$ yield. PEGBPQDs were completely soluble in aqueous media and showed two characteristic $n-\pi^{*}$ and $\pi-\pi^{*}$ transition peaks of CCDP at 257 and $360 \mathrm{~nm}$, respectively, confirming the mussel-inspired attachment of CA-PEG on BPQD (Figure 4a). Then, the PL spectra of PEG-BPQDs were examined by increasing the excitation wavelength from 325 to $500 \mathrm{~nm}$. Similar to the blue emission of the pristine BPQDs, PEG-BPQDs showed blue PL emission centered at $428 \mathrm{~nm}$ under UV light $(365 \mathrm{~nm})$. However, PEG-BPQDs presented irradiation-dependent PL emission behavior in vivid contrast to the irradiationindependent PL emission of pristine BPQDs (Figure 4b). Whereas the origin of dramatic change in the $\mathrm{PL}$ emission behavior of BPQDs after the surface functionalization is not fully understood in this stage, this excitation-dependent PL emission is widely observed in CQDs or CDs. In the case of CQDs or CDs, single molecular level PL study has shown that this excitation dependency of PL emission originates from either the ensemble emission of CDs showing different emission color $^{54}$ or overlaid emission of each $\mathrm{CD}$ having different fluorophores. ${ }^{55,56}$

The morphological features of PEG-BPQDs were examined by TEM (the SI, Figure S11), AFM (the SI, Figure S12), and dynamic light scattering (DLS) (the SI, Figure S13), respectively. The lateral sizes of PEG-BPQDs were 40-60 and $\sim 78 \mathrm{~nm}$ in TEM and DLS, respectively. The increased lateral size measured from the DLS might originate from the solvated CA-PEG shells on the surface of BPQDs. AFM showed the thickness of PEG-BPQDs ranging from 25 to 40 $\mathrm{nm}$. From all of these characterizations, it is clear that PEG$\mathrm{BPQDs}$ have increased lateral size due to the presence of outer CA-PEG shells on the BPQD cores. However, this core-shell image was not revealed from the TEM or HRTEM analysis probably due to the similar electron density both in polymeric shells and BPQD cores. The prepared PEG-BPQDs showed a high dispersion stability in aqueous media and also in aqueous buffer solution (PBS or FBS) under ambient condition. Negligible decrease in fluorescence intensity less than $1 \%$ was observed with the exposure by day light up to 14 days (the SI, Figure S14). QY of PEG-BPQDs in aqueous solution was 4.4\%, which is slightly smaller than the QY $(\sim 5 \%)$ of pristine BPQDs in chloroform. The facile dispersion of PEG-BPQDs in the aqueous media together with the unique irradiation-dependent PL emission makes PEG-BPQDs a useful multicolor fluorescence bioimaging agent. The in vitro cell viability of PEG-BPQDs was investigated toward either MDCK normal cells or $\mathrm{KB}$ tumor cells. Up to the concentration of $1 \mathrm{mg} / \mathrm{mL}$ (1000 ppm), PEG-BPQD demonstrated a high cell viability of $>91 \%$ toward both cells, indicating that PEG-BPQDs can be utilized as the less-toxic fluorescence bioimaging agent (Figure 4c). The low toxicity of PEG-BPQDs might rely mainly on the presence of biocompatible PEG segments on the surface of BPQDs. Then, KB tumor cells were incubated in the presence of PEG-BPQD ( 1 or $10 \mathrm{mg} / \mathrm{mL}$, respectively), and the cultured $\mathrm{KB}$ cells were investigated by confocal laser scanning microscopy. Distinctly, the KB cells showed the evident blue fluorescence at their corresponding excitation wavelengths, indicating the facile cellular uptake and in vitro fluorescence cell imaging performance of PEG-BPQD (Figure 4d).

\section{CONCLUSIONS}

In conclusion, we have first demonstrated that stable blueemitting BPQDs can be prepared in chloroform by a simple sonication-assisted liquid-phase exfoliation of BP flakes in the ambient atmosphere. The prepared BPQDs were highly dispersible in organic solvents including toluene and methanol, regardless of the polarity. Furthermore, simple mussel-inspired surface functionalization of BPQDs with PEG afforded watersoluble and blue-emitting PEG-BPQDs, showing very low cytotoxicity together with excellent fluorescence cellular imaging capability.

\section{EXPERIMENTAL SECTION}

Preparation of BPQDs. The preparation of soluble BPQDs is based on the sonication-assisted liquid-phase exfoliation of bulk BP crystal. The storage of BP crystal (flake form) has been totally attempted inside the nitrogen-purged glovebox throughout the experiments to minimize continual surface oxidation of pristine BPs. As a representative example, $10 \mathrm{mg}$ of BP was delivered out of glovebox and immediately sonicated in $10 \mathrm{~mL}$ of chloroform by tip-sonicator in ambient atmosphere using Q500 sonicator (500 W, Qsonica, LLC). To screen solvent effect on the exfoliation of BPs, all of the liquid-phase exfoliation setups were attempted simultaneously using a tipsonicator attached with a 24 multitip accessory to minimize the effects of other experimental parameters such as temperature and humidity. The power amplitude of sonicator was fixed at $80 \%$ and continuous $5 \mathrm{~s}$ on/10 s off operation of sonicator was attempted to minimize the buildup of heat in the dispersing media. After $1 \mathrm{~h}$ of sonication, nonexfoliated bigger BP particles were removed from the dispersion of the BPQDs by centrifugation at either 2500 or $7500 \mathrm{rpm}$, resulting in the synthesis of soluble BPQDs showing a strong blue-light emission.

At first, aqueous-soluble catechol-grafted PEG (CA-PEG) was prepared by the reaction between 2 -chloro- $3^{\prime}, 4^{\prime}$-dihydroxyacetophenone (CCDP) and poly( $N$-isopropyl acrylamide)grafted poly(ethylene glycol) (PEG-g-PNIPAM) by the quaternization reaction of CCDP on the nitrogen moieties of PNIPAM. The number of catechol groups in single CA-PEG polymer was estimated as 15 from the ${ }^{1} \mathrm{H}$ NMR spectrum of the prepared CA-PEG. ${ }^{50}$ At second, the mussel-inspired surface functionalization of BPQDs with CA-PEG was attempted in a mixed solvent of EtOH and tris-buffered silane (TBS, pH 9.0). After purification by centrifugation and freeze drying, the PEGmodified BPQD (PEG-BPQD) was successfully obtained with $>90 \%$ yield. PEG-BPQDs were completely soluble in aqueous media and showed two characteristic $n-\pi^{*}$ and $\pi-\pi^{*}$ 
transition peaks of CCDP at 257 and $360 \mathrm{~nm}$, respectively, confirming the mussel-inspired attachment of CA-PEG on BPQD.

Preparation of PEG-BPQDs. PEG (Mn of XXX, $0.4 \times$ $\left.10^{-3} \mathrm{M}\right)$, dimethyl acrylamide (DMA) $\left(15 \times 10^{-3} \mathrm{M}\right)$, and NIPAAm $\left(80 \times 10^{-3} \mathrm{M}\right)$ were dissolved in $120 \mathrm{~mL}$ of anhydrous toluene and the initiator $t$-butylperoxybenzoate (1.0 $\left.\times 10^{-3} \mathrm{M}\right)$ was added to the solution to produce PEG-gPNIPAM. The mixture was then stirred for $24 \mathrm{~h}$ at $140{ }^{\circ} \mathrm{C}$. The product was purified by precipitation in hexane after rotary evaporation and dried in a vacuum. To prepare CA-PEG through quaternization, PEG- $g$-PNIPAM $\left(M_{n} 30940\right.$ Da measured by gel permeation chromatography, $0.1 \times 10^{-3} \mathrm{M}$ ) and CCDP $\left(1.5 \times 10^{-3} \mathrm{M}\right)$ were dissolved in $50 \mathrm{~mL}$ of $1: 1$ ethanol in a $250 \mathrm{~mL}$ flask. The mixture was stirred for $24 \mathrm{~h}$ at $70{ }^{\circ} \mathrm{C}$. After stirring, the solvent was evaporated in a rotary evaporator and precipitated using cold diethyl ether, producing CA-PEG as white powders after vacuum drying. To synthesize PEG-BPQDs, $0.2 \mathrm{~g}$ of CA-PEG and $4 \mathrm{mg}$ of BPQD were dispersed in the mixture solvent of $9 \mathrm{~mL}$ of ethanol and $1 \mathrm{~mL}$ of TBS buffer ( $\mathrm{pH}$ 9.0). After magnetic stirring for $24 \mathrm{~h}$, centrifugation at $4000 \mathrm{rpm}$ for 5 min produced blue-emitting supernatant and black precipitate. The supernatant solution was collected and freeze-dried under reduced pressure, producing PEG-BPQD powders with 93.7 wt \% mass yield based on the initial BPQD mass.

In Vitro Cell Viability of PEG-BPQD. The cytotoxicity was measured using the [3-(4,5-dimethylthiazol-2-yl)-2,5-diphenyltetrazolium bromide] MTT assay method. $200 \mathrm{~mL}$ of $\mathrm{KB}$ cells and MDCK cells, at a density of $2 \times 10^{5}$ cells $/ \mathrm{mL}$, was placed in each well of a 96-well plate and incubated for $24 \mathrm{~h}$ at $37^{\circ} \mathrm{C}$ in a humidified $5 \% \mathrm{CO}_{2}$ atmosphere. To determine the cellular viability, a stock solution of PEG-BPQDs was dissolved in Roswell Park Memorial Institute medium at a concentration of $1 \mathrm{mg} / \mathrm{mL}$, and the stock solution was diluted to $0.01 \mathrm{mg} / \mathrm{mL}$. The medium was removed, and the cells were treated with different concentrations of PEG-BPQDs. The cells were then incubated for another $24 \mathrm{~h}$. The medium containing PEGBPQDs was then replaced with $180 \mathrm{~mL}$ fresh medium and 20 $\mathrm{mL}$ of a stock solution containing $15 \mathrm{mg}$ of MTT in $3 \mathrm{~mL}$ PBS and incubated for another $4 \mathrm{~h}$. Finally, the medium was removed and $200 \mathrm{~mL}$ MTT solubilizing agent was added to the cell and mixed thoroughly for $15 \mathrm{~min}$. Absorbance was measured at $570 \mathrm{~nm}$ wavelength using a microplate reader (Varioskan Flash, Thermo Electron Corporation). The relative cell viability was measured by comparing the control 96-well containing only the cell.

In Vitro Cell Imaging. The cellular uptakes of PEG$\mathrm{BPQDs}$ were analyzed using confocal imaging. The $\mathrm{KB}$ cells were plated over a cover slide on an eight-well plate $200 \mathrm{~mL}$ at a density of $1 \times 10^{5}$ cells $/ \mathrm{mL}$ per well and incubated for $24 \mathrm{~h}$ at $37{ }^{\circ} \mathrm{C}$ in a humidified $5 \%$ of $\mathrm{CO}_{2}$ atmosphere. The cells were then treated with PEG-BPQDs at 1 or $10 \mathrm{mg} / \mathrm{mL}$ for $30 \mathrm{~min}$ in fresh culture media, respectively. The cells were then washed with PBS several times to remove the unbound PEG-BPQDs and then examined at $20 \times$ magnification using an LSM510 confocal laser scanning microscope.

Characterization. UV-vis spectra were obtained from the UV-vis spectrometer of Hewlett-Packard. PL spectra were obtained from L550B luminescence spectrometer of PerkinElmer. TEM and HRTEM images were recorded with FEI Tecnai T20 at an accelerating voltage of $200 \mathrm{kV}$. AFM topographic images were obtained from XE-100 model of PSIA.
FTIR spectra were obtained from Nicolet IR 200. XPS data were obtained from Multilab 2000 of Thermo Scientific. Raman spectra were obtained from LabRAM high-resolution dispersive Raman microscope of Horiba Jobin Yvon using a $633 \mathrm{~nm} \mathrm{He}-$ $\mathrm{Ne}$ laser beam. The NMR spectra were recorded using an Avance digital $400 \mathrm{MHz}$ Bruker Spectrometer (Germany). $\zeta$ Potential measurements were made with a Zetasizer ELS Z (Otsuka Electronics, South Korea). Confocal fluorescent images were taken with a confocal laser microscopy system (Confocal, LSM510, Carl Zeiss, Oberkochen 73447, Germany). The measurement of QY of BPQDs and PEG-BPQDs was attempted by the reference point method typically attempted for estimating QYs of other blue-emitting fluorescent (nano)materials by a comparison with quinine sulfate ( $\mathrm{QY}$ of 0.54 in $0.1 \mathrm{M} \mathrm{H}_{2} \mathrm{SO}_{4}$ ).

\section{ASSOCIATED CONTENT}

\section{Supporting Information}

The Supporting Information is available free of charge on the ACS Publications website at DOI: 10.1021/acsomega.7b01058.

Twenty-four organic solvents in various solvent categories with different surface tensions and molecular weights were tested toward the liquid-phase exfoliation of BP flake (Table S1); highest PL emission intensity was observed for the BPQD dispersion in chloroform at 437 $\mathrm{nm}$; irradiation-independent PL emission intensity was observed when DMAc was used as the organic solvent during the liquid-phase exfoliation of BP; irradiationindependent PL emission intensity was observed when pyridine was observed similarly to the PL emission behavior of certain CQDs or CDs; Raman spectroscopy characterization of four different samples; digital photo images of PS and PS/BPQD composite film (2 wt \% BPQDs); fluorescence spectra of PS/BPQD composite film ( 2 wt \% BPQDs) with the excitation wavelength of 300 and $377 \mathrm{~nm}$; positive $\zeta$ potential was repeatedly observed for the chloroform dispersion of the BPQDs; ${ }^{31} \mathrm{P}$ NMR spectroscopy was recorded for both the BPQD dispersion (blue line) and pristine BP (black line) measured in $\mathrm{CDCl}_{3}$ with $85 \%$ phosphoric acid as the reference; ${ }^{13} \mathrm{C}$ NMR spectrum of BPQD clearly shows carbon peaks at 29.9 and $27.1 \mathrm{ppm} ;{ }^{1} \mathrm{H}$ NMR spectrum of the prepared CA-PEG; TEM image of PEG-BPQDs on carbon-coated $\mathrm{Cu}$ grid; AFM images of BPQDs on $\mathrm{Si}$ wafer; size distribution profile of PEG-BPQDs $(0.5 \mathrm{mg} /$ $\mathrm{mL}$ in water) measured by DLS; relative fluorescence intensity of PEG-BPQDs $(1 \mathrm{mg} / \mathrm{mL})$ in $2 \%$ aqueous buffer solutions (Figures S1-S14) (PDF)

\section{AUTHOR INFORMATION}

\section{Corresponding Authors}

*E-mail: kimsy@kaist.ac.kr (S.Y.K.).

*E-mail: parkchem@ut.ac.kr (S.Y.P.).

*E-mail: in1@ut.ac.kr (I.I.).

ORCID $\odot$

Soo-Jin Park: 0000-0002-6350-6135

Jihoon Lee: 0000-0002-7736-027X

Sang Youl Kim: 0000-0003-4106-1996

Sung Young Park: 0000-0002-0358-6946

Insik In: 0000-0001-7852-1162 


\section{Author Contributions}

OY.P. and M.L. contributed equally to this work.

\section{Notes}

The authors declare no competing financial interest.

\section{ACKNOWLEDGMENTS}

M.L. is the recipient of Global Ph.D. Fellowship (NRF2014H1A2A1021021). This research was supported by the Ministry of Trade, Industry, and Energy (MOTIE) and Korea Institute for Advancement of Technology (KIAT) through the Research and Development for Regional Industry (R0005303), the Radiation Technology R\&D program through the NRF funded by the Ministry of Science, ICT \& Future Planning (NRF-2017M2A2A6A01019289), the Basic Science Research Program through the National Research Foundation of Korea (NRF) funded by the Ministry of Education (2015R1D1A3A01020192), and International Joint Technology Development Project (N0002123) through the Ministry of Trade, Industry, and Energy (MI, Korea) and the Korea Institute for KIAT.

\section{REFERENCES}

(1) Zhu, S.; Song, Y.; Zhao, X.; Shao, J.; Zhang, J.; Zhang, B. The photoluminescence mechanism in carbon dots (graphene quantum dots, carbon nanodots and polymer dots): current state and future perspective. Nano Res. 2015, 8, 355-381.

(2) Lim, S. Y.; Shen, W.; Gao, Z. Carbon quantum dots and their applications. Chem. Soc. Rev. 2015, 44, 362-381.

(3) Wang, Z.; Zeng, H.; Sun, L. Graphene quantum dots: versatile photoluminescence for energy, biomedical, and environmental applications. J. Mater. Chem. C 2015, 3, 1157-1165.

(4) Peng, F.; Su, Y.; Zhong, Y.; Fan, C.; Lee, S.; He, Y. Silicon Nanomaterials Platform for Bioimaging, Biosensing, and Cancer Therapy. Acc. Chem. Res. 2014, 47, 612-623.

(5) Cheng, X.; Lowe, S. B.; Reecec, P. J.; Gooding, J. J. Colloidal silicon quantum dots: from preparation to the modification of selfassembled monolayers (SAMs) for bio-applications. Chem. Soc. Rev. 2014, 43, 2680-2700.

(6) Ji, X.; P, F.; Zhong, Y.; Su, Y.; Jiang, X.; Song, C.; Yang, L.; Chu, B.; Lee, S.; He, Y. Highly Fluorescent, Photostable, and Ultrasmall Silicon Drug Nanocarriers for Long-Term Tumor Cell Tracking and In-Vivo Cancer Therapy. Adv. Mater. 2015, 27, 1029-1034.

(7) Little, W.; Karatutlu, A.; Bolmatov, D.; Trachenko, K.; Sapelkin, A. V.; Cibin, G.; Taylor, R; Mosselmans, F.; Dent, A. J.; Mountjoy, G. Structural origin of light emission in germanium quantum dots. Sci. Rep. 2014, 4, No. 7372.

(8) Robel, I.; Shabaev, A.; Lee, D. C.; Schaller, R. D.; Pietryga, J. M.; Crooker, S. A.; Efros, A. L.; Klimov, V. I. Temperature and MagneticField Dependence of Radiative Decay in Colloidal Germanium Quantum Dots. Nano Lett. 2015, 15, 2685-2692.

(9) van Noorden, R. The Trials of New Carbon. Nature 2011, 469, $14-16$.

(10) Chen, D.; Feng, H.; Li, J. Graphene Oxide: Preparation, Functionalization, and Electrochemical Applications. Chem. Rev. 2012, 112, 6027-6053.

(11) Chen, R. Apparent stretched-exponential luminescence decay in crystalline solids. J. Lumin. 2003, 102-103, 510-518.

(12) Lange, S.; Schmidt, P.; Nilges, T. $\mathrm{Au}_{3} \mathrm{SnP}_{7} @ B$ lack Phosphorus: An Easy Access to Black Phosphorus. Inorg. Chem. 2007, 46, 40284035.

(13) Nilges, T.; Kersting, M.; Pfeifer, T. A fast low-pressure transport route to large black phosphorus single crystals. J. Solid State Chem. 2008, 181, 1707.

(14) Nagao, M.; Hayashi, A.; Tatsumisago, M. J. All-solid-state lithium secondary batteries with high capacity using black phosphorus negative electrode. J. Power Sources 2011, 196, 6902-6905.
(15) Sun, Z.; et al. Ultrasmall Black Phosphorus Quantum Dots: Synthesis and Use as Photothermal Agents. Angew. Chem., Int. Ed. 2015, 54, 11526-11530.

(16) Zhu, C.; et al. Ultrafast Preparation of Black Phosphorus Quantum Dots for Efficient Humidity Sensing. Chem. Eur. J. 2016, 22, 7357-7362.

(17) Gu, W.; et al. Black Phosphorus Quantum Dots as the Ratiometric Fluorescence Probe for Trace Mercury Ion Detection Based on Inner Filter Effect. ACS Sens. 2017, 2, 576-582.

(18) Sun, L. Q.; et al. Electrochemical Activity of Black Phosphorus as an Anode Material for Lithium-Ion Batteries. J. Phys. Chem. C 2012, $116,14772-14779$

(19) Deng, Y.; Luo, Z.; Conrad, N. J.; Liu, H.; Gong, Y.; Najmaei, S.; Ajayan, P. M.; Lou, J.; Xu, X.; Ye, P. D. Black Phosphorus-Monolayer MoS2 van der Waals Heterojunction p-n Diode. ACS Nano 2014, 8, $8292-8299$.

(20) Fiori, G.; Bonaccorso, F.; Iannaccone, G.; Palacios, T.; Neumaier, D.; Seabaugh, A.; Banerjee, S. K.; Colombo, L. Electronics based on two-dimensional materials. Nat. Nanotechnol. 2014, 9, 768779 .

(21) Li, L.; Yu, Y.; Ye, G. J.; Ge, Q.; Ou, X.; Wu, H.; Feng, D.; Chen, X. H.; Zhang, Y. Black phosphorus field-effect transistors. Nat. Nanotechnol. 2014, 9, 372-377.

(22) Zhang, S.; Yang, J.; Xu, R.; W, F.; Li, W.; Ghufran, M.; Zhang, Y.; Yu, Z.; Zhang, G.; Qin, Q.; Lu, Y. Extraordinary Photoluminescence and strong Temperature/Angle Dependent Raman Responses in Few-Layer Phosphorene. ACS Nano 2014, 8, 95909596.

(23) Low, T.; Rodin, A. S.; Carvalho, A.; Jiang, Y.; Wang, H.; Xia, F.; Castro Neto, A. H. Tunable optical properties of multilayers black phosphorus thin films. Phys. Rev. B 2014, 90, No. 075434.

(24) Zhang, X.; Xie, H.; Liu, Z.; Tan, C.; Luo, Z.; Li, H.; Lin, J.; Sun, L.; Chen, W.; Xu, Z.; Xie, L.; Huang, W.; Zhang, H. Black Phosphorus Quantum Dots. Angew. Chem., Int. Ed. 2015, 54, 3653-3657.

(25) Yasaei, P.; Kumar, B.; Foroozan, T.; Wang, C.; Asadi, M.; Tuschel, D.; Indacochea, J. E.; Klie, R. F.; Khojin, A. S. High-Quality Black Phosphorus Atomic Layers by Liquid-Phase Exfoliation. Adv. Mater. 2015, 27, 1887-1892.

(26) Kang, J.; Wood, J. D.; Wells, S. A.; Lee, J.; Liu, X.; Chen, K.; Hersam, M. C. Solvent Exfoliation of Electronic-Grade, TwoDimensional Black Phosphorus. ACS Nano 2015, 9, 3596-3604.

(27) Lee, M.; Roy, A. K.; Jo, S.; Choi, Y.; Chae, A.; Kim, B.; Park, S. Y.; In, I. Exfoliation of black phosphorus in ionic liquids. Nanotechnology 2017, 28, No. 12.

(28) Hernandez, Y.; Nicolosi, V.; Lotya, M.; Blighe, F. M.; Sun, Z.; De, S.; Mcgovern, I. T.; Holland, B.; Byrne, M.; Gunko, Y. K.; Boland, J. J.; Niraj, P.; Duesberg, G.; Krishnamurthy, S.; Goodhue, R.; Hutchison, J.; Scardaci, V.; Ferrari, A. C.; Coleman, J. N. High-yield production of graphene by liquid-phase exfoliation of graphite. Nat. Nanotechnol. 2008, 3, 563-568.

(29) Coleman, J. N. Liquid Exfoliation of Defect-Free Graphene. Acc. Chem. 2013, 46, 14-22.

(30) Roy, A. K.; Park, B.; Lee, K. S.; Park, S. Y.; In, I. Boron nitride nanosheets decorated with silver nanoparticles through musselinspired chemistry of dopamine. Nanotechnology 2014, 25, No. 445603.

(31) Zhao, C.; Xu, Z.; Wang, H.; Wei, J.; Wang, W.; Bai, X.; Wang, E. Carbon-Doped Boron Nitride Nanosheets with Ferromagnetism above Room Temperature. Adv. Funct. Mater. 2014, 24, 5985-5992.

(32) Yeon, Y.; Lee, M.; Kim, S. Y.; Lee, J.; Kim, B.; Park, B.; In, I. Production of quasi-2D graphene nanosheets through the solvent exfoliation of pitch-based carbon fiber. Nanotechnology 2015, 26, No. 375602.

(33) Jiang, K.; Sun, S.; Zhang, L.; Lu, Y.; Wu, A.; Cai, C.; Lin, H. Red, Green, and Blue Luminescence by Carbon Dots: Full-Color Emission Tuning and Multicolor Cellular Imaging. Angew. Chem., Int. Ed. 2015, $54,5360-5363$.

(34) Roy, A. K.; Kim, S.; Paoprasert, P.; Park, S.; In, I. Preparation of biocompatible and antibacterial carbon quantum dots derived from 
resorcinol and formaldehyde spheres. RSC Adv. 2015, 5, 3167731682 .

(35) Zhong, Y.; Peng, F.; Wei, X.; Zhou, Y.; Wang, J.; Jiang, X.; Su, Y.; Su, S.; Lee, S.; He, Y. Microwave-Assisted Synthesis of Biofunctional and Fluorescent Silicon Nanoparticles Using Proteins as Hydrophilic Ligands. Angew. Chem., Int. Ed. 2012, 51, 8485-8489.

(36) Zhong, Y.; Peng, F.; Bao, F.; Wang, S.; Ji, X.; Yang, L.; Su, Y.; Lee, S.; He, Y. Large-Scale Aqueous Synthesis of Fluorescent and Biocompatible Silicon Nanoparticles and Their Use as Highly Photostable Biological Probes. J. Am. Chem. Soc. 2013, 135, 83508356.

(37) Castellanos-Gomez, A.; Vicarelli, L.; Prada, E.; Island, J. O.; Narasimha-Acharya, K. L.; Blanter, S. I.; Groenendijk, D. J.; Buscema, M.; Steele, G. A.; Alvarez, J. V.; Zandbergen, H. W.; Palacios, J. J.; van der Zant, H. S. Isolation and characterization of few-layer black phosphorus. 2D Mater. 2014, 1, No. 025001.

(38) Wood, J. D.; Wells, S. A.; Jariwala, D.; Chen, K.; Cho, E.; Sangwan, V. K.; Liu, X.; Lauhon, L. J.; Marks, T. J.; Hersam, M. C. Effective Passivation of Exfoliated Black Phosphorus Transistors against Ambient Degradation. Nano Lett. 2014, 14, 6964-6970.

(39) Wang, G.; Pandey, R.; Karna, S. P. Phosphorene oxide: stability and electronic properties of a novel two-dimensional material. Nanoscale 2015, 7, 524-531.

(40) Island, J. O.; Steele, G. A.; van der Zant, H. S. J.; CastellanosGomez, A. Environmental instability of few-layer black phosphorus. $2 D$ Mater. 2015, 2, No. 011002.

(41) Tran, V.; Soklaski, R.; Liang, Y.; Yang, L. Tunable Band Gap and Anisotropic Optical Response in Few-layer Black Phosphorus. Phys. Rev. B 2014, 89, No. 235319.

(42) Liang, L.; Wang, W.; Lin, B.; Sumpter, G.; Meunier, V.; Pan, M. Electronic Bandgap and Edge Reconstruction in Phosphorene Materials. Nano Lett. 2014, 14, 6400-6406.

(43) Das, S.; Zhang, W.; Demarteau, M.; Hoffmann, A.; Dubey, M.; Roelofs, A. Tunable Transport Gap in Phosphorene. Nano Lett. 2014, 14, 5733-5739.

(44) Sun, Y.; Zhou, B.; Lin, Y.; Wang, W.; Shiral Fernando, K. A.; Pathak, P.; et al. Quantum-Sized Carbon Dots for Bright and Colorful Photoluminescence. J. Am. Chem. Soc. 2006, 128, 7756-7757.

(45) Shen, J.; Zhu, Y.; Yang, X.; Li, C. Graphene quantum dots: emergent nanolights for bioimaging, sensors, catalysis and photovoltaic devices. Chem. Commun. 2012, 48, 3686-3699.

(46) Nilges, T.; Kersting, M.; Pfeifer, T. A fast low-pressure transport route to large black phosphorus single crystals. J. Solid State Chem. 2008, 181, 1707-1711.

(47) Lu, W.; Nan, H.; Hong, J.; Chen, Y.; Zhu, C.; Liang, Z.; Ma, X.; Ni, Z.; Jin, C.; Zhang, Z. Plasma-assisted fabrication of monolayer phosphorene and its Raman characterization. Nano Res. 2014, 7, 853859.

(48) Shen, Z.; Sun, S.; Wang, W.; Liu, J.; Liu, Z.; Yu, J. C. A blackred phosphorus heterostructure for efficient visible-light-driven photocatalysis. J. Mater. Chem. A 2015, 3, 3285-3288.

(49) Ramireddy, T.; Xing, T.; Rahman, M. M.; Chen, Y.; Dutercq, Q.; Gunzelmann, D.; Glushenkov, A. M. Phosphorus-carbon nanocomposite anodes for lithium-ion and sodium-ion batteries. J. Mater. Chem. A 2015, 3, 5572-5584.

(50) Kang, S. M.; Park, S.; Kim, D.; Park, S. Y.; Ruoff, R. S.; Lee, H. Simultaneous Reduction and Surface Functionalization of Graphene Oxide by Mussel-Inspired Chemistry. Adv. Funct. Mater. 2011, 21, $108-112$.

(51) Li, S. C.; Chu, Li; Gong, X.; Diebold, U. Hydrogen Bonding Controls the Dynamics of Catechol Adsorbed on a $\mathrm{TiO}_{2}(110)$ Surface. Science 2010, 328, 882-884.

(52) Liu, Y.; Ai, K.; Lu, L. Polydopamine and Its Derivative Materials: Synthesis and Promising Applications in Energy, Environmental, and Biomedical Fields. Chem. Rev. 2014, 114, 5057-5115.

(53) Kim, S. M.; Park, Y. H.; Seo, S. W.; Park, C. P.; Park, S. Y.; In, I. Mussel-Inspired Immobilization of Catalysts for Microchemical Applications. Adv. Mater. Interfaces 2015, 2, No. 1500174.
(54) Das, S. K.; Liu, Y.; Yeom, S.; Kim, D. Y.; Richards, C. I. SingleParticle Fluorescence Intensity Fluctuations of Carbon Nanodots. Nano Lett. 2014, 14, 620-625.

(55) Choi, Y.; Jo, S.; Chae, A.; Kim, Y. K.; Park, J. E.; Lim, D.; Park, S. Y.; In, I. Simple Microwave-Assisted Synthesis of Amphiphilic Carbon Quantum Dots from $\mathrm{A}_{3} / \mathrm{B}_{2}$ Polyamidation Monomer Set. ACS Appl. Mater. Interfaces 2017, 9, 27883-27893.

(56) Kang, E. B.; Sharker, S. M.; In, I.; Park, S. Y. Pluronic mimicking fluorescent carbon nanoparticles conjugated with doxorubicin via acidcleavable linkage for tumor-targeted drug delivery and bioimaging. $J$. Ind. Eng. Chem. 2016, 43, 150-157. 